\title{
Termite digestomes as a potential source of symbiotic microbiota for lignocelluloses degradation: a review
}

\begin{abstract}
Termites thrive in great abundance in terrestrial ecosystems and the symbiotic gut microbiota play important roles in digestion of lignocelluloses and nitrogen metabolism. Termites are excellent models of biocatalysts as they inhabit dense microbes in their guts that produce digestive enzymes to decompose lignocelluloses and convert it to end products such as sugars, hydrogen, and acetate. Different of digestive system between lower and higher termites which lower termites dependent on their dual decomposing system, consisting of termite's own cellulases and gut's protists. Higher termites decompose cellulose using their own enzymes, because of the absence of symbiotic protists. Termite gut prokaryotes efficiently support lignocelluloses degradation. In this review, a brief overview of recent experimental works, development and commercialization is discussed. Significant progress has been made to isolate cellulolytic strains from termites and optimise the digestion efficiency of cellulose. Future perspective should emphasize the isolation of cellulolytic strains from termites, genetically modifying or immobilization of the microbes which produce the desired enzyme and thus benefits on the microbiology and biotechnology.
\end{abstract}

Keyword: Termite; Lignocelluloses; Polysaccharides; Microbes; Bacteria; Flagellate 